\title{
UN RECURSO DIDÁCTICO BASADO EN EL USO DE UN SISTEMA DE PREGUNTAS ABIERTAS SOPORTADO POR TECNOLOGÍA
}

\section{A DIDACTIC RESOURCE BASED ON THE USE OF AN OPEN-ENDED QUESTIONS SYSTEM SUPPORTED BY TECHNOLOGY}

\author{
Ricardo Elías Valdivia Pinto; rvaldivi@academicos.uta.cl \\ Patricia Vilma Castillo Ochoa; pcastillo@uta.cl \\ Universidad de Tarapacá (Chile)
}

\begin{abstract}
RESUMEN
Este artículo presenta la descripción e implementación de una dinámica de preguntas abiertas como recurso didáctico orientado al desarrollo del dominio y la compresión -tanto de conceptos como de contenidos- por parte de los estudiantes de educación superior.

La propuesta se centra en una variación de las dinámicas basadas en la votación sobre preguntas de selección múltiple. Esta dinámica propone el uso de preguntas abiertas para generar interacciones entre los participantes del curso como también con los conocimientos a lo largo del mismo. La dinámica está soportada por una plataforma que apoya la gestión del profesor en relación a la selección del estudiante como también en las estadísticas de comportamiento de los participantes en el curso.
\end{abstract}

PALABRAS CLAVE: Aprendizaje en el aula, Sistemas de Respuestas en Clases, Constructivismo, Recurso didáctico

\begin{abstract}
This article presents the description and implementation of a dynamic of questions open as a teaching resource aimed at the development of the domain and compression -both concepts and content- by students in higher education. The proposal focuses on a variation of dynamics based on the voting on multiple-choice questions. This dynamic proposes the use of open-ended questions to generate interactions among the participants of the course as well as along the same knowledge. The dynamic is supported by a platform that supports the management of teacher in relation to the selection of the student as well as the statistics of the behavior of the participants in the course.
\end{abstract}

KEYWORDS: Classroom face-to-face learning, Class response system, Constructivism, Teaching resource. 


\section{INTRODUCCIÓN}

\subsection{Problema}

El uso de recursos tecnológicos como parte del proceso de enseñanza implica un desafío para el docente, dado que requiere de un trabajo coordinado entre tecnología, pedagógica y aspectos curriculares, todos ellos orientados a la construcción de aprendizajes de parte de los estudiantes.

Uno de los recursos que han sido más implementados es el denominado sistema de respuestas en clases. Este sistema permite que los estudiantes respondan en clases distintas preguntas que previamente el docente debe ingresar en una plataforma.

El problema se origina ya que hasta ahora las respuestas de los estudiantes solo podían ser de tipo cerrada, lo que limitaba la profundización de los contenidos abordados. Por esta razón surgió la necesidad de generar un sistema que permita profundizar en las respuestas de parte del estudiante, y a la vez registrar las intervenciones permitiendo así disponer de información para el seguimiento de los estudiantes y su avance académico.

A partir del enfoque constructivista del proceso de enseñanza y aprendizaje, el rol docente debiera potenciar el pensamiento crítico, creativo, reflexivo además de generar un aprendizaje estratégico (Monereo \& Pozo, 2003, Carretero, M, 1999, Díaz, F., \& Hernández, G. 2004). En este contexto el uso efectivo de recursos digitales flexibles que apunten al logro de competencias desde una mirada colaborativa y de seguimiento mutuo, permitiría contribuir a la labor docente, por un lado, como factor que va a permitir al estudiante comprender y abordar diferentes tipos de problemas y por otro ayudar al profesor con datos útiles para el seguimiento de su grupo clase.

\subsection{Revisión de la literatura}

La globalización de la educación y de la formación implica nuevas formas de aprender dentro de una educación expansiva en vez de restringida; multidireccional en vez de unidireccional; donde la coordinación de acciones es la prioridad. A partir de ello se hace necesario analizar y diseñar recursos que contribuyan a renovar la enseñanza en la Educación Superior, desde una perspectiva didáctica a fin de inaugurar espacios de innovación curricular, con intenciones educativas que favorezcan la construcción de conocimientos y competencias requeridas (Senge, Kleiner, Roberts, Ross, Roth \& Smith 1999).

El aprendizaje como proceso requiere actuaciones de parte de los agentes en la que intervienen dos factores: conocimientos previos y la actividad externa o interna que el estudiante realice al respecto (Carretero, 1999). Desde la concepción constructivista de la enseñanza y del aprendizaje se desprende la práctica social y socializadora por esencia.

La propuesta de una dinámica de preguntas abiertas involucra la realización de actividades asociadas a la discusión y resolución de problemas lo que permite a los y las estudiantes a la discusión sobre el proceso de deducción que han utilizado. Por lo que se logra promover la construcción del conocimiento mientras los y las estudiantes interactúan en el marco de un proceso reflexivo guiado por el profesor (Powers \& Powers, 1999). 
Entre las ventajas del uso de preguntas de selección múltiple (Roberts, 2006) se indica que proveen de rápida retroalimentación, pueden ser automáticamente evaluadas, y pueden almacenarse en bases de datos de preguntas para ser reutilizadas. Entre las desventajas, se argumenta que en su construcción se requiere de un esfuerzo considerable, que solo pueden evaluar conocimiento y memorización, y no consideran aspectos de análisis y creatividad, resultando en que los estudiantes adoptan una actitud superficial al aprendizaje.

Los estudios llevados a cabo en el uso de estos sistemas, conocidos genéricamente como sistemas de respuesta en clases (Classroom Response Systems, CRS) (Roschelle, 2003) o sistemas de comunicación en clases (Classroom Communication Systems, CCS) (Beatty, 2004) han mostrado que el rol de la tecnología es valioso al interior de la clase (Woodford \& Bancroft, 2001). La tecnología provee anonimidad, rápida recolección de respuestas y la habilidad de producir una visualización compartida la cual mejora el reconocimiento de posiciones comunes. La tecnología entonces actúa como un catalizador, produciendo un cambio en el clima de la clase, la pedagogía y el aprendizaje resultante (Miao, Holst, Holmer, Freschutz, \& Zentel, 2000).

\subsection{Propósito}

Dar a conocer la aplicación de un recurso didáctico que intenciona en la interacción y comprensión de los contenidos de parte de los y las estudiantes en el aula (Valdivia \& Castillo, 2007)

\section{METODOLOGÍA}

Como una variante de los sistemas CRS, la dinámica descrita en este trabajo, considera un conjunto de características diferenciadoras orientadas a mejorar los problemas que involucra tanto la implementación como el uso de ellas. En particular, (a) se basa en el uso de preguntas abiertas, (b) el tiempo se extiende más allá de la clase, cubriendo el desarrollo de todo el semestre, y finalmente, (c) incorpora la evaluación como un factor de organización e incentivo en la dinámica.

El uso de preguntas abiertas pretende por un lado ampliar el espacio de posibles preguntas a ser realizadas a los y las estudiantes y por otro fortalecer tanto sus capacidades de reflexión y crítica, como sus habilidades comunicativas. Las preguntas realizadas clase a clase, incentivan a los estudiantes para que participen activamente en la construcción de su conocimiento. La evaluación, basada en un proceso de acumulación de puntos: (a) permite que la dinámica proceda como un continuo (los estudiantes son informados de su rendimiento y esperan informados la próxima actividad), (b) incentiva la participación (los estudiantes desean optar a una mejor evaluación en cada clase), (c) requiere que el profesor organice la participación considerando las necesidades de cada estudiante.

Al comienzo del curso, el profesor debe establecer una "meta" semestral basada en la cantidad de preguntas que debiese realizar, esto con el fin de que optimizar las posibilidades que cada estudiante tendrá de participar de la actividad durante el transcurso del semestre. El sistema debe apoyar claramente esta característica, visualizando la 
participación y rendimiento de los estudiantes, ayudando al profesor a tomar mejores decisiones en cuanto a "que" estudiante seleccionar al momento de plantear una pregunta. Para el soporte de la dinámica propuesta, se ha seguido la tendencia en el uso de sistemas web para el soporte de dinámicas CRS como Kahoot. (Pintor et al, 2014) Estos sistemas permiten sustituir el original uso de tecleras (clickers) por la actual disponibilidad de dispositivos móviles (tablets o smartphones) como dispositivos de votación.

La dinámica sigue las siguientes etapas:

1. Realización de una pregunta por parte del Profesor. En ese momento la participación queda abierta a los estudiantes a través de sus dispositivos móviles.

2. Participación de los Estudiantes en la Pregunta quienes deciden su participación en la pregunta para lo cual dispone de un botón que le provee el sistema (ver Figura 1).

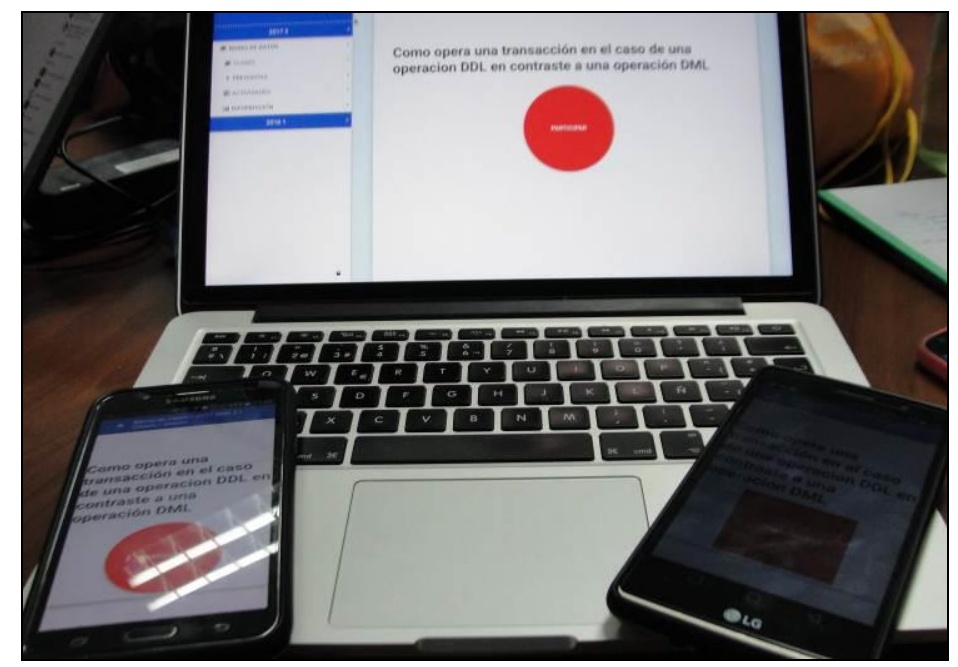

Figura 1: Botón de participación sobre diferentes dispositivos

3. Despliegue de la lista de Participación. El profesor debe esperar, de acuerdo a su criterio, un tiempo adecuado para que los estudiantes se inscriban para participar. Durante este tiempo, la lista de alumnos que participan de la pregunta se va actualizando en la proyección.

La lista de alumnos entrega información importante para la selección del estudiante que responderá la pregunta. Criterios como posición, preguntas contestadas correcta y erradamente durante el semestre y la clase, y veces que ha participado sin ser seleccionado son mostradas para que el profesor tome una adecuada decisión en la selección del alumno (ver Figura 2).

Una vez que el profesor lo estima conveniente, cierra el proceso de participación a los estudiantes. 


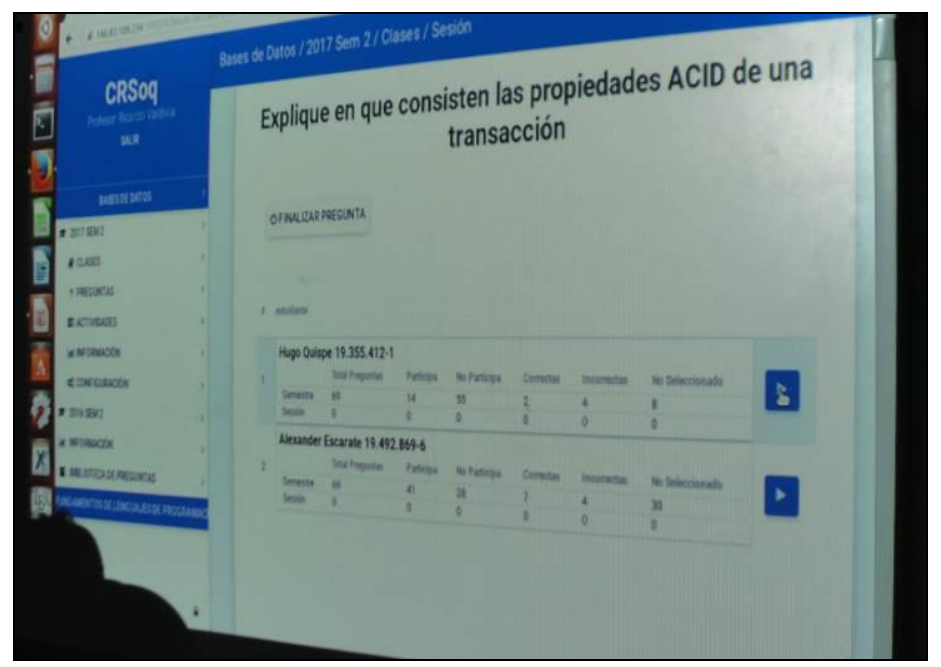

Figura 2: Lista de participación

4. Selección del Estudiante. El profesor selecciona a través del sistema al estudiante que responderá la pregunta, para esto dispone de la información indicada anteriormente.

5. Respuesta de los Estudiantes. El estudiante seleccionado debe dar su respuesta a la clase, y luego esperar la evaluación por parte del profesor. En este momento, el profesor puede generar una conversación abierta para tomar una decisión al respecto (ver Figura 3).

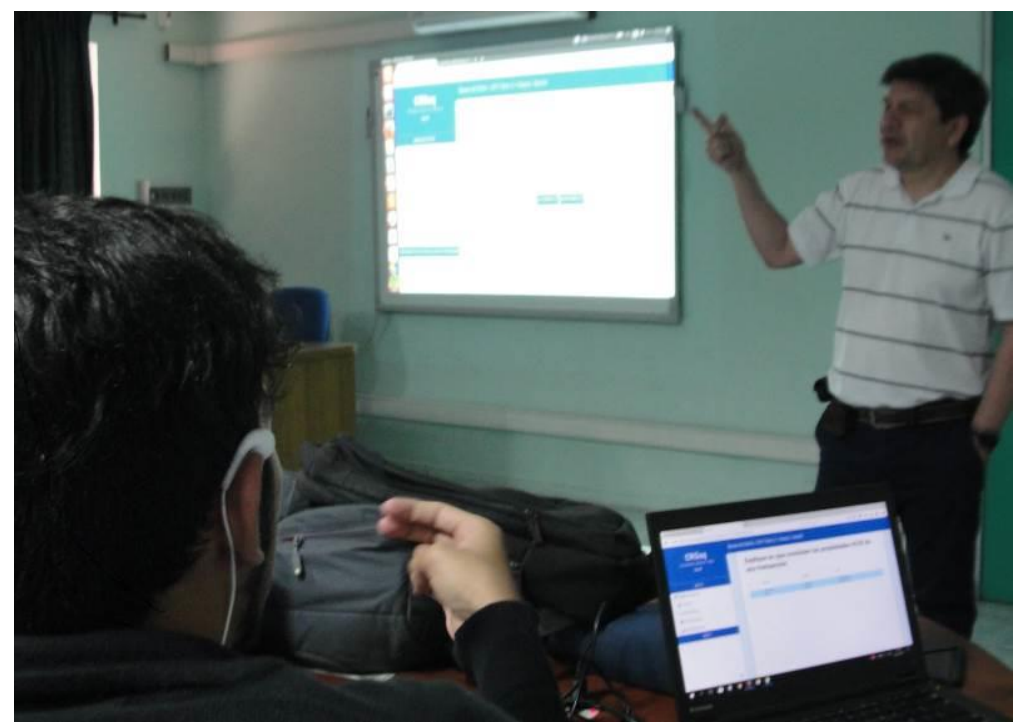

Figura 3: Estudiante argumentando su respuesta

6. Evaluación de la Respuesta. El profesor determina si la respuesta es correcta entregando el puntaje al estudiante a través del sistema, el cual lo registra. En caso de ser incorrecta, puede seleccionar otro estudiante y volver a la etapa anterior, o simplemente dar por cerrada la pregunta sin ganadores.

7. Cierre de la Sesión El proceso puede continuar con nuevas preguntas, recomenzando desde la etapa 1, o dar por concluida la sesión, la que es cerrada en el sistema. 
Actualmente, el sistema desarrollado CRSoq (Classroom Response System Open-ended Questions) incluye la gestión de cursos, nómina de estudiantes y base de preguntas de forma de soportar adecuadamente la dinámica.

\section{RESULTADOS}

En este punto presentamos las gráficas que resultan de la aplicación del sistema CRSoq.

Durante el semestre, el profesor y los estudiantes tienen acceso a información asociada al estado de la dinámica, de forma que puedan ajustar su participación en la misma.

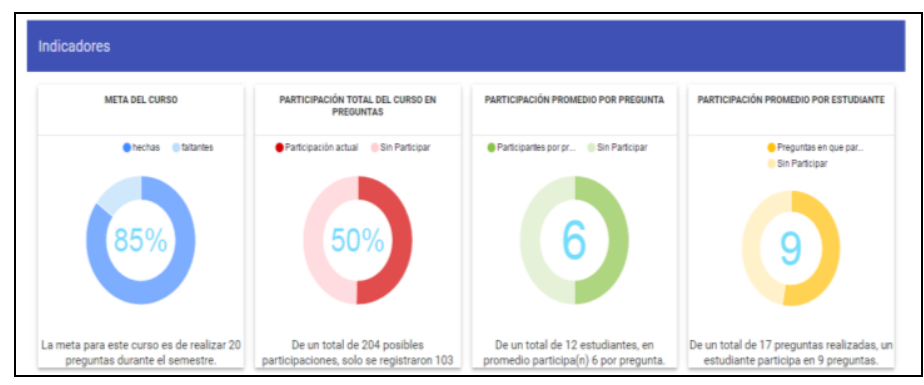

Figura 4: Indicadores de la dinámica.

Los indicadores de la Figura 4 describen cuatro aspectos relevantes del curso El primer indicador muestra el grado de avance de la meta del curso. El segundo indicador muestra el grado de participación del curso. El tercer indicador muestra cual es el promedio de estudiantes que participan por pregunta. Finalmente, el cuarto indicador señala cual es el promedio de participación de un estudiante.

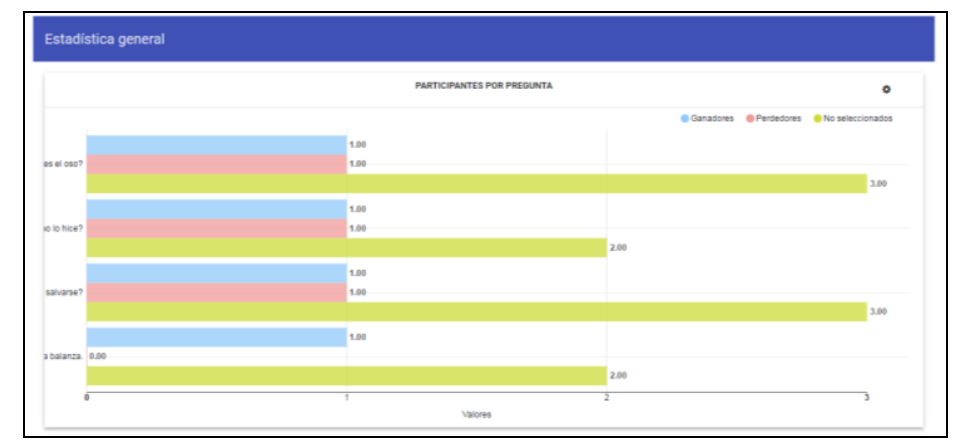

Figura 5: Gráfico de participación por pregunta.

El gráfico de la Figura 5 permite visualizar los participantes por pregunta, ganadores, perdedores y los que no fueron seleccionados. 


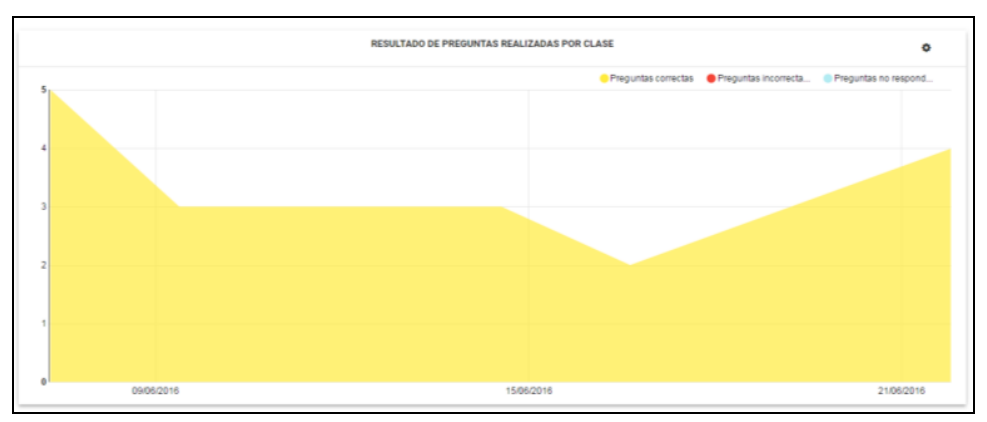

Figura 6: Gráfico de preguntas realizadas clase a clase.

El gráfico de la Figura 6 permite visualizar el número de preguntas realizadas clase a clase, ya sea si se respondieron de forma correcta, incorrecta o no tuvieron respuesta.

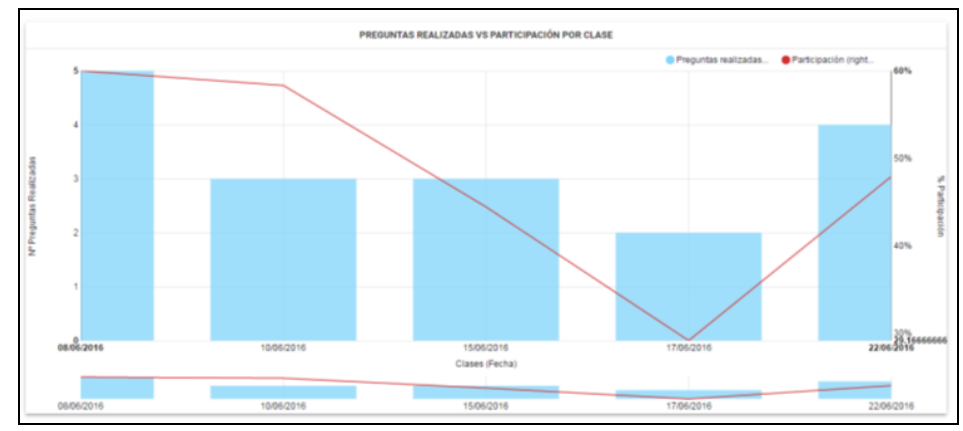

Figura 7: Gráfico de preguntas realizadas versus la participación por clases.

El gráfico de la figura 7 muestra la cantidad de preguntas realizadas clase a clase contrastado con el porcentaje de participación de los estudiantes.

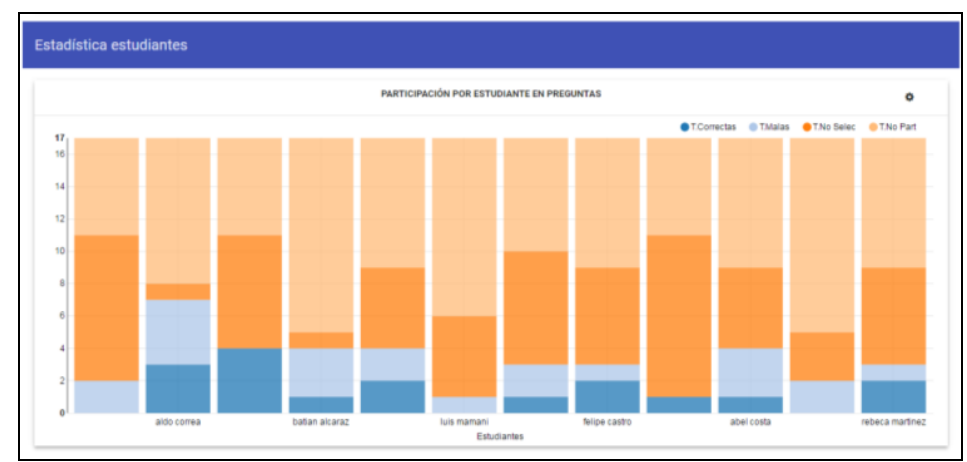

Figura 8: Gráfico de participación por estudiante.

En el gráfico de la Figura 8 es posible observar la participación de cada estudiante, ya sea el total de preguntas correctas, o en las que no fue seleccionado. 


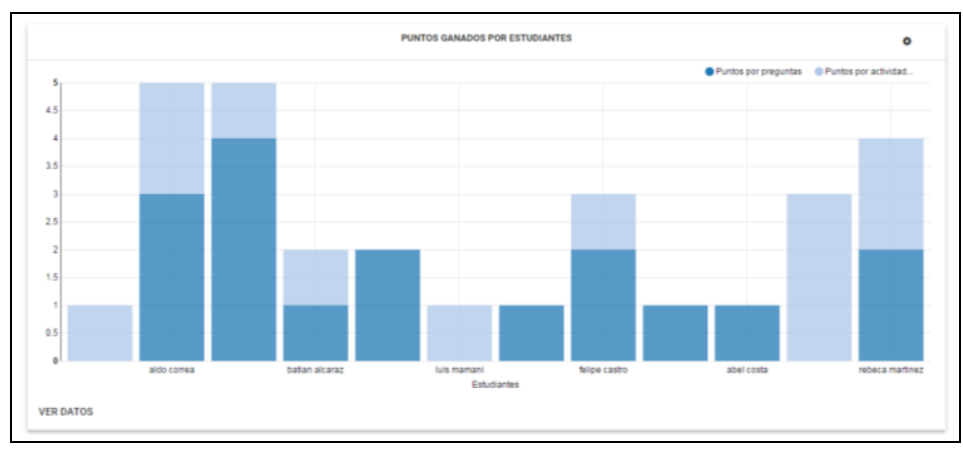

Figura 9: Gráfico de puntos ganados por los estudiantes.

En el gráfico de la Figura 9 el profesor puede visualizar la cantidad de puntos obtenidos por cada estudiante durante el semestre.

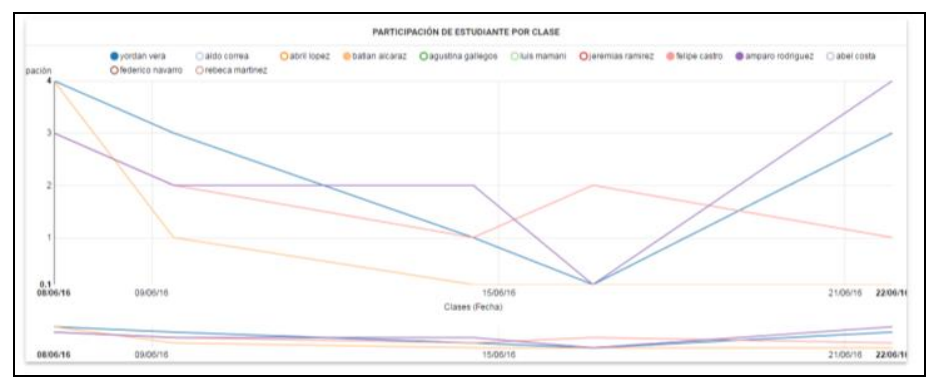

Figura 10: Gráfico de participación por cada estudiante clase a clase.

En la Figura 10 se presenta un gráfico con la curva de participación de cada estudiante. Así, el profesor puede analizar el desempeño de cada estudiante clase a clase.

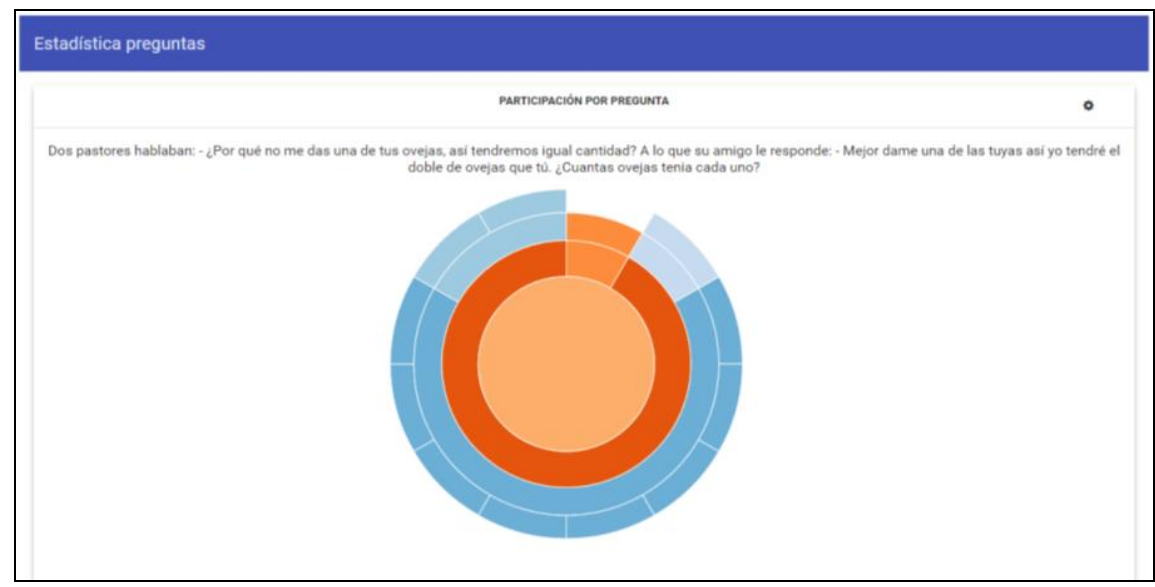

Figura 11: Gráfico de detalle por pregunta.

Finalmente, en el gráfico de la figura 11 es posible contar con una estadística por pregunta. En éste gráfico interactivo, el profesor puede ver el detalle por cada pregunta, considerando: el número de participantes, quienes participaron y quien fue el ganador en la pregunta 
En general, estos gráficos permiten ajustar diferentes filtros, para obtener reportes más detallados.

\section{DISCUSIÓN Y CONCLUSIONES}

Los sistemas CRS han evolucionado desde los tiempos de Classtalk (Dufresne et al, 1996), uno de los primeros sistemas de este tipo, incorporando el uso de nuevas tecnologías o variaciones en la dinámica original. La tecnología de soporte ha evolucionado desde el uso de clickers wireless, considérese el caso de H-ITT (Siau, Sheng, \& Nah, 2006), al soporte de smartphones y tablets, vease Kahoot (Pintor et al, 2014). La dinámica ha sido modificada para introducir nuevas ideas, como CANA (Valdivia, Ochoa, \& Nussbaum, Modeling a Collaborative Answer Negotiation Activity Using IMS-Based Learning Design., 2009) que enfatiza la interacción en pequeños grupos de estudiantes, o THM (Lucke, Keyssner, \& Dunn, 2013) que introduce elementos de aula invertida. Nuestra propuesta es una variación que modifica radicalmente la naturaleza de estos sistemas al introducir el uso de preguntas abiertas. La dinámica que es soportada por el sistema mantiene, sin embargo, gran parte de los beneficios propios de los sistemas CRS, en particular, la posibilidad de reutilizar preguntas, la interactividad de los estudiantes y la retroalimentación al profesor. Algunos aspectos pierden validez, como la participación conjunta de la clase en el proceso de responder la pregunta original, pero el proceso de discusión es más rico, las preguntas pueden involucrar un nivel de complejidad mayor y abre la posibilidad de ampliar la discusión al resto de la clase.

El empleo de preguntas abiertas durante la clase, su incentivo y evaluación pretende generar situaciones que mejoren el proceso de enseñanza aprendizaje en la clase. A través del debate de ideas, se espera crear un espacio donde el profesor pueda reforzar y corregir conceptos que requieren de un proceso de discusión al interior de la clase, y que además necesitan de un proceso de continua retroalimentación (ver Figura 12).

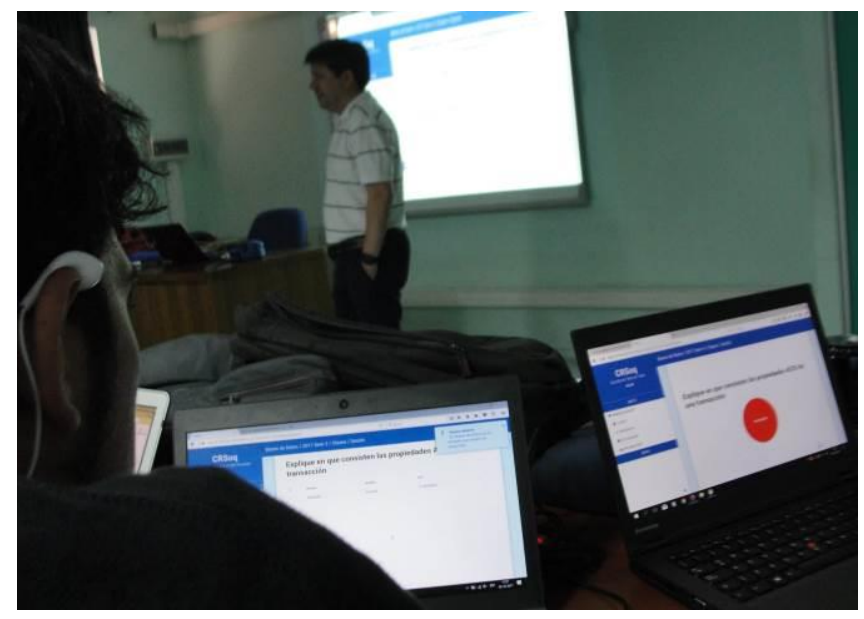

Figura 12: Clase basada en la dinámica en desarrollo 
La dinámica variante CRS, permite al estudiante expresar sus argumentos al aula por lo que se espera que mejore sus habilidades expositivas, de pensamiento crítico y reflexivo.

El sistema CRSoq está pensado como un sistema de soporte, contribuyendo así a la gestión y evaluación del proceso educativo, facilitando la toma decisiones de parte del profesor como de los estudiantes ya que permite visualizar los niveles de participación y de rendimiento del grupo curso.

El uso del sistema propuesto, facilita la obtención de información inmediata en relación a los logros obtenidos de los y las estudiantes por lo que se logra retroalimentar oportunamente.

Adicionalmente, el profesor logra visualizar el funcionamiento de una pregunta en particular en la historia del curso, permitiéndole ajustar o descartar aquellas que no funcionan de acuerdo a lo esperado.

\section{REFERENCIAS}

Beatty, I. (2004). Transforming Student Learning with Classroom Communication Systems. EDUCAUSE Center for Applied Research, 2004(3). Obtenido de https://library.educause.edu/resources/2004/2/transforming-student-learnin

Carretero, M. (1999). Constructivismo y Educación. Buenos Aires: Paidós.

Díaz, F., \& Hernández, G. (2004). Estrategias docentes para un aprendizaje significativo. Una interpretación constructivista. México: McGraw- Hill.

Dufresne, W., Gerace, W., Leonard, W., Mestre, J., \& Wenk, L. (1996). Classtalk: A Classroom Communication System for Active Learning. Journal of Computing in Higher Education, 7(2), 3-47.

López, B. (2008). Estilos de docencia y evaluación de los profesores universitarios y su influencia sobre los modos de aprender de sus estudiantes. . Revista Española De Pedagogía, 66(241), 425-445.

Lucke, T., Keyssner, U., \& Dunn, P. (2013). The Use of a Classroom Response System to More Effectively Flip the Classroom. Frontiers in Education Conference. IEEExplore Digital Library. Obtenido de http://ieeexplore.ieee.org

Mari, J. (s.f.). Manual de redacción científica. Universidad de Puerto Rico, Departamento de Biología, Mayagüez, Puerto Rico.

Miao, Y., Holst, S., Holmer, T., Freschutz, J., \& Zentel, P. (2000). An Activity-Oriented Approach to Visually Structured Knowledge Representation for Problem-Based Learning in Virtual Learning Environments, Designing Cooperative Systems. The Use of Theories an. Proceedings of the 5th International Conference on the Design of Cooperative Systems. 58, págs. 303-318. Francia: Frontiers in Artificial Intelligence and Applications. 
Monereo, C., \& Pozo, J. (2003). La cultura educativa en la universidad: nuevos retos para profesores y alumnos. La universidad ante la nueva cultura educativa. Enseñar y aprender para la autonomía, 15-30. doi:DOI 10.13140/2.1.5069.2168

Pintor, E., Gargantilla, P., Herreros, B., \& López, M. (2014). Kahoot en docencia. Una Alternativa Práctica a los Clickers. XI Jornadas Internacionales de Innovación Universitaria: Educar para Transformar.

Powers, D., \& Powers, K. (1999). Making Sense of Teaching Methods in Computing Education. Frontiers in Education Conference. San Juan de Puerto Rico. doi:10.1109/FIE.1999.839224

Roberts, T. (2006). The Use of Multiple Choice Test for Formative and Summative Assessment. Conferences in Research in Practice in Information Technolo. Tasmania: Eighth Australasian Computing Education Conference .

Roschelle, J. (2003). Unlocking the Learning Value of Wireless Mobile Devices. ournal of Computer Assisted Learning, 19(3), 260-272. Obtenido de https://www.sri.com/work/publications/unlocking-learning-value-wireless-mobiledevices

Senge, P., Kleiner, A., Roberts, C., Ross, R., Roth, G., \& Smith, B. (1999). La danza del cambio: los desafíos de mantener el impulso en las organizaciones de aprendizaje. Mueva York: Currency Doubleday. doi:10.1002 /pfi.4140380511

Siau, K., Sheng, H., \& Nah, F. F.-H. (2006). Use of a Classroom Response System to Enhance Classroom Interactivity. IEEE Transactions on Education, 49(3).

Valdivia, R., \& Castillo, P. (2007). Una Dinámica de Preguntas Abiertas como Recurso de Aprendizaje en el Aula. XX Congreso Internacional EDUTEC. Santiago de Chile.

Valdivia, R., Ochoa, S., \& Nussbaum, M. (2009). Modeling a Collaborative Answer Negotiation Activity Using IMS-Based Learning Design. IEEE Transactions on Education, 52(3), 375-384.

Woodford, K., \& Bancroft, P. (2001). Using multiple choice questions effectively in information tecnology education. University of Technology, School of Software Engineering and Data Communications Queensland. Recuperado el 23 de Enero de 2017, de http://citeseerx.ist.psu.edu/viewdoc/download?doi=10.1.1.86.3166\&rep=rep1\&typ $\underline{e=p d f}$

\section{Para referenciar este artículo:}

Valdivia-Pinto, R., Vilma Ochoa, P. (2017). Un curso didáctico basado en el uso de un sistema de preguntas abiertas soportado por tecnología. EDUTEC, Revista electrónica de Tecnología Educativa, 62. Recuperado de: http://dx.doi.org/10.21556/edutec.2017.62.1015 